INPLASY

PROTOCOL

To cite: He et al. Fat embolism syndrome: a case report, literature review and pooled analysis. Inplasy protocol 2020100039. doi:

10.37766/inplasy2020.10.0039

Received: 11 October 2020

Published: 12 October 2020

Corresponding author:

Zuo wen He

hezuowen@hust.edu.cn

Author Affiliation:

Tongji Hospital, Tongji Medical College, Huazhong University of Science and Technology, Wuhan, China.

Support: NSFC (81790624, 81900341).

Review Stage at time of this submission: The review has not yet started.

Conflicts of interest:

None.

\section{Fat embolism syndrome: a case report, literature review and pooled analysis}

He, Z1; Shi, Z2; Li, C3; Ni, L4; Sun, Y5; Wang, Y6; Wang, D7.

Review question / Objective: Participants: Patients diagnosed with FES, Interventions: Glucocorticoid treatment, Comparisons: Patients without glucocorticoid treatment, Outcomes: In-hospital death, Study design: Pooled analysis of 136 individual retrieved from 126 case reports from June. 25th, 2010 to June. 25th, 2020.

Condition being studied: We further performed pooled analysis of 136 such cases retrieved from 126 isolated reports. This study firstly analyzes clinical features of FES, compares survival and non-survival patients, evaluates treatment patterns and risk factors associated with death.

INPLASY registration number: This protocol was registered with the International Platform of Registered Systematic Review and Meta-Analysis Protocols (INPLASY) on 12 October 2020 and was last updated on 12 October 2020 (registration number INPLASY2020100039).

\section{INTRODUCTION}

Review question / Objective: Participants: Patients diagnosed with FES, Interventions: Glucocorticoid treatment, Comparisons: Patients without glucocorticoid treatment, Outcomes: In-hospital death, Study design:
Pooled analysis of 136 individual retrieved from 126 case reports from June. 25th, 2010 to June. 25th, 2020.

Rationale: Fat embolism syndrome (FES) is a very rare and life-threatening clinical syndrome. Few clinical features and 
outcome patterns of FES have been acknowledged.

Condition being studied: We further performed pooled analysis of 136 such cases retrieved from 126 isolated reports. This study firstly analyzes clinical features of FES, compares survival and non-survival patients, evaluates treatment patterns and risk factors associated with death.

\section{METHODS}

Search strategy: An extensive review of the English language literatures was performed in PubMed. literatures were obtained by searching on databases of PubMed with keywords of "fat embolism syndrome", with limitation of "case report, 10 years publication, human species, English languages". Studies with incomplete data, irrelevant information and non-Englsih were excluded.

Participant or population: We included all cases older than 14 years old with confirmed FES described in the literature the last decades and with complete information about general information, etiologies, clinical presentations, diagnosis patterns, auxiliary examinations and treatments.

Intervention: FES patients treated with glucocorticoid.

Comparator: FES patients treated without glucocorticoid.

Study designs to be included: This systematic review and pooled-analysis followed the Preferred Reporting Items for Systematic reviews and Meta-Analyses for Individual Patient Data.

Eligibility criteria: There is no RCT, metaanalysis and systemic review to evaluate the role of glucocorticoid treatment in mortality of FES. Therefore, we included: 1. all cases older than 14 years old with confirmed FES described in the English literature the last decades. 2. Cases with complete information about general information, etiologies, clinical presentations, diagnosis patterns, auxiliary examinations and treatments.

Information sources: All the information were obtained from Pubmed database.

Main outcome(s): The main outcome in this study was in-hospital death.

Quality assessment / Risk of bias analysis: There is no RCT, meta-analysis and systemic review to evaluate the role of glucocorticoid treatment in mortality of FES. Therefore, we plan to collect all cases of FES with complete information in the last decades from Pubmed database, The quality of case reports will be assessed with the Grading of Recommendation, Assessment, Development and Evaluation (GRADE), with study and report quality categorized as high, moderate, low, or very low.

Strategy of data synthesis: There is no RCT, meta-analysis and systemic review to evaluate the role of glucocorticoid treatment in mortality of FES. Therefore, we plan to collect all cases of FES with complete information in the last decades from Pubmed database, and then we plan to extract data standardly by tabulating the following information: sex, age, comorbidity, etiology, fracture sites, clinical presentations, auxiliary examination performance, diagnosis mode, treatments and prognosis. Two investigators will conduct the literature search independently to verify data accuracy and completeness, with a third reviewer resolving any uncertainties.

Subgroup analysis: Compares survival and non-survival patients.

Sensibility analysis: We will conduct sensitivity analyses for the main outcome.

Language: English.

Country(ies) involved: China.

Keywords: Fat embolism syndrome, Pulmonary embolism, Diffused alveolar 
d a mage, Respiratory failure, Glucocorticoid, Pooled analysis.

Contributions of each author:

Author 1 - Zuo wen He.

Author 2 - Zeqi Shi.

Author 3 - Chenze Li.

Author 4 - Li Ni.

Author 5 - Yang Sun.

Author 6 - Yan Wang.

Author 7 - Dao Wen Wang. 